\title{
El consumo de tabaco como automedicación de depresión/ansiedad entre los jóvenes: resultados de un estudio con método mixto
}

\section{Smoking as a form of self-medication for depression or anxiety in young adults: results of a mixed-methods study}

\author{
Natàlia Carceller-Maicas*; Santiago Ariste**; Angel Martínez-Hernáez**; María-Antonia \\ Martorell-Poveda***; Martín Correa-Urquiza***; Susan M. DiGiacomo**** \\ * Departamento de Antropología, Universitat Rovira i Virgili; ** Departamento de Psicología, Universitat Rovira i Virgili; \\ *** Departamento de Enfermería, Universitat Rovira i Virgili; **** Departamento de Antropología, Universitat Rovira i Virgili \\ y University of Massachusetts at Amherst.
}

\section{Resumen}

El consumo de tabaco y los problemas de salud mental de tipo depresivo/ansioso son dos fenómenos que suelen iniciarse en la adolescencia y juventud con cierta co-ocurrencia. Ambos fenómenos guardan una relación bidireccional que, en el caso de los jóvenes, la hipótesis de automedicación parece explicar de forma más exhaustiva que otras opciones. El objetivo de este estudio es explorar la relación entre consumo de tabaco, síntomas de depresión y ansiedad y percepción de los jóvenes sobre el uso del tabaco como forma de automedicación. Para ello se seleccionaron 105 jóvenes (17-21 años) de un estudio sociológico longitudinal previo considerando tres grupos de participantes: 1) sujetos con diagnóstico de depresión/ ansiedad en anteriores oleadas, 2) sujetos con malestar depresivo/ ansioso autopercibido y sin diagnóstico previo y 3) sujetos control. Se aplicó un cuestionario mixto cuantitativo/cualitativo de consumo de substancias y las escalas BDI-II de depresión y GHQ en su cribado de ansiedad-depresión, así como la escala MISS (Mannheim Interview on Social Support). Los resultados indican que los sujetos afectados por síntomas de depresión/ansiedad en la adolescencia se inician más tarde en el consumo de cigarrillos, pero los que son fumadores alegan principalmente como motivo la automedicación. La asociación entre consumo habitual de tabaco y niveles de depresión de la escala BDI no fue significativa para la muestra general, pero sí para los jóvenes varones (OR: 6,22, IC95\%, 1,06-36,21, p=.042). Las iniciativas antitabáquicas dirigidas a los jóvenes deben considerar los problemas de malestar emocional y el consumo de tabaco como forma de automedicación.

Palabras clave: consumo de tabaco, depresión, ansiedad, automedicación, adolescentes, jóvenes.

\begin{abstract}
Tobacco use and mental health problems in the depression/anxiety spectrum often begin in adolescence as co-occurring phenomena. Epidemiologically, the relationship between them is bidirectional, but in the case of young people it appears to be explained best by the unidirectional self-medication hypothesis. The aim of this study is to explore the relationship between tobacco use, symptoms of depression or anxiety, and the perception of adolescents and young adults concerning tobacco use as a form of self-medication. A sample of 105 young people between the ages of 17 and 21 years was selected from a longitudinal sociological study to create three groups of participants: 1) subjects with a previous diagnosis of depression or anxiety; 2) subjects with self-perceived but undiagnosed distress compatible with depression or anxiety; 3) and a group of control. A mixed quantitative/qualitative questionnaire on substance consumption was administered, as well as the BDI-II depression scale, the GHQ anxiety and depression scales, and the MISS (Mannheim Interview on Social Support) scale. The final results show that the subjects experiencing symptoms of depression or anxiety in adolescence start smoking later than subjects in the control group, and those who smoke give selfmedication as the main reason for doing so. The association between habitual tobacco use and BDI scores for depression was not statistically significant for the sample as a whole, only for the male participants (OR: 6,22, IC95\%, 1,06-36,21, p=.042). Anti-smoking campaigns targeting young people should take into consideration their use of tobacco as a form of self-medication for emotional distress.

Key words: tobacco use, depression, anxiety, self-medication, adolescents, young adults.
\end{abstract}

Recibido: febrero 2013; Aceptado: julio 2013

Enviar correspondencia a:

Angel Martínez-Hernáez. Dept. Antropología, Universitat Rovira i Virgili (Campus Catalunya). Avinguda de Catalunya, 35.43002 Tarragona. Telf. 639731 685. E-mail: angel.martinez@urv.cat 


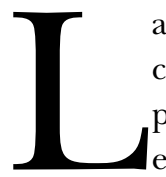

a adolescencia y la juventud son etapas vitales asociadas con el debut en el consumo de substancias psicoactivas, incluyendo el tabaco, y también con el incremento de problemas de salud mental. La mayor parte de los trastornos mentales aparecen antes de los 25 años de edad y, entre ellos, los del espectro depresivo y ansioso adquieren un carácter central por sus implicaciones clínicas y sociales, como suicidio, desajuste social, cronicidad, consumo de substancias psicoactivas y trastorno mental severo en la vida adulta (Birchwood y Singh, 2013). Se calcula que la prevalencia de vida entre los 13 y los 18 años es de 14,3\% para los trastornos del estado de ánimo y de 31,9\% para los trastornos de ansiedad (Merikangas et al., 2010). Esta estimación tiene en cuenta exclusivamente los casos de trastorno franco, a los que debemos adicionar los estados de malestar subclínicos (Balázs et al., 2013).

Los síntomas depresivos y/o ansiosos y el consumo de tabaco son dos fenómenos que están asociados entre sí, pero cuya relación ha sido objeto de polémica. Por un lado, existe evidencia de una asociación bidireccional en diversos estudios (Audrain-McGovern, Rodriguez y Kassel, 2009; Steuber y Danner, 2006) y una revisión sistemática ha mostrado que sufrir depresión predice el hábito tabáquico (1.41, IC 95\%: $1.21,1.63)$ y, a la vez, que fumar predice la aparición de síntomas depresivos (1.73 IC 95\%: 1.32, 2.40) (Chaiton, Cohen, Loughlin y Rehm, 2009). Por otro lado, sin embargo, desconocemos la relación causal que se establece entre estos dos fenómenos, fundamentalmente por la existencia de un conocimiento aún incompleto sobre las bases de los trastornos depresivos y ansiosos y de las adicciones, así como por la diversidad de factores involucrados que pueden actuar de mediadores y que presumiblemente oscilan entre el campo de la genética y el terreno del comportamiento social. En este último, destacan las influencias contextuales de la familia y el grupo de iguales, que han sido ampliamente exploradas en la literatura y que parecen mostrar un peso relevante en la vulnerabilidad a la iniciación en el hábito tabáquico, al incremento del consumo de tabaco (Calleja y Aguilar, 2008) y a su co-ocurrencia con los síntomas de depresión y ansiedad (Rohde, Lewinsohn, Brown, Gau, y Kahler, 2003).

Algunas investigaciones han reforzado las hipótesis de la automedicación (self-medication) (Audrain-McGovern et al., 2009) y del stress-coping-model (Hrubá y Zaloudíková, 2010) como modelos explicativos de la asociación entre depresión/ansiedad y hábito tabáquico, indicando que los individuos afectados por trastornos o malestares de este espectro fumarían para resolver y/o regular sus estados emocionales. Es sabido que los receptores de acelticolina nicotínicos (nAChRs) son el principal objetivo de la nicotina. Mediante los nAChRs, la nicotina tiene un efecto en neurotransmisores como la serotonina que modula la respuesta a la depresión y el stress (Mienur y Picciotto, 2010). Tanto la nicotina como los agentes nicotínicos reducen los síntomas depresivos, explicando el fenómeno de la autome- dicación y generando comorbilidad (Weinberger, MCkee, Picciotto y Mazure, 2011). En consonancia con este argumento, algunas investigaciones indican que los adolescentes con depresión mayor presentan una frecuencia más elevada de consumo de tabaco y mayor dependencia nicotínica en comparación con los no afectados. La existencia de síntomas subclínicos de depresión, por su parte, predice la iniciación temprana al consumo, la progresión a ser fumador habitual y el aumento en el número de cigarrillos fumados diariamente (Escobedo, Reddy y Giovino, 1998; Fergusson, Goodwin y Horwood, 2003).

En oposición a la hipótesis de la automedicación, algunas investigaciones se han orientado en la dirección inversa; esto es: el consumo habitual de tabaco sería un factor de riesgo del debut en la depresión y/o en la ansiedad (Choi, Patten, Gillin, Kaplan, y Pierce, 1997); Klungsoyr, Nygard, Sorensen y Sandanger, 2006; Lyons et al., 2008). Estos trabajos enfatizan el efecto del hábito tabáquico en los niveles serotoninérgicos, como es el caso de algunos estudios recientes que muestran que el consumo semanal de cigarrillos activa las mismas regiones cerebrales que el consumo diario $(\mathrm{Ru}-$ binstein et al., 2011). Una tercera hipótesis es, sin duda, que tanto el consumo de tabaco como los síntomas depresivo/ ansiosos respondan a terceros factores, como es el caso de la desigualdad social (Fergusson, Horwood y Boden, 2007). No obstante, y al margen de su posible asociación, los dos fenómenos aquí debatidos adquieren por sí solos relevancia por sus implicaciones en la salud de los jóvenes, incluyendo los efectos a largo plazo.

A pesar de la diversidad de investigaciones realizadas, en la literatura no predominan los estudios desarrollados con un enfoque mixto (cuantitativo/cualitativo) y que incluyan los motivos percibidos por los jóvenes sobre el carácter de su consumo como mecanismo de automedicación o resolución de sus malestares. El objetivo de esta investigación es explorar con un modelo mixto (cuantitativo-cualitativo) la relación entre los estados de depresión/ansiedad, el consumo de tabaco y la percepción de los jóvenes sobre los motivos de fumar en una submuestra extraída de un estudio sociológico longitudinal realizado previamente en Cataluña.

\section{Método}

\section{Diseño del estudio y selección de la muestra}

Los jóvenes de este estudio fueron reclutados del Panel de Familias e Infancia (PFI), un estudio sociológico longitudinal en cuatro oleadas diseñado por el Instituto de Infancia y Mundo Urbano (CIIMU) (Institut d'Infància i Món Urbà 2012; Julià, Escapa, Marí-Klose y Marí-Klose, 2012; Martínez-Hernáez et al. 2012). Esa investigación se inició en 2006 con una muestra representativa de 3004 adolescentes residentes en Cataluña nacidos entre los años 1990 y 1993, ambos incluidos, y fue incorporando nuevas cohortes cada año. En ese estudio se recogía información sobre estados 
de ánimo negativos mediante una escala autoadministrada (años 2007 y 2008) y sobre la existencia de un diagnóstico de depresión/ansiedad (años 2006 y 2010), además de una diversidad de factores sobre consumo de substancias psicoactivas, sociabilidad y factores económicos, escolares y familiares.

La selección de nuestra muestra se llevó a cabo considerando tres grupos de participantes: a) con depresión/ ansiedad diagnosticada en la $1^{\mathrm{a}}$ o $4^{\mathrm{a}}$ oleada del PFI según el relato de sus progenitores (la pregunta incidía sobre si existía un diagnóstico profesional de depresión/ansiedad); b) con malestar autopercibido de tipo depresivo en las oleadas $2^{\mathrm{a}}$ y $3^{\mathrm{a}}$ pero sin diagnóstico de depresión/ansiedad; y c) sin diagnóstico ni malestar autopercibido. La selección se efectuó a través de la técnica "Propensity Matching Score", obtenida sobre la base de predictores observables mediante un análisis de regresión logística. Se seleccionaron 50 jóvenes para cada uno de los tres grupos $(\mathrm{N}=150)$. El número de seleccionados respondió a un criterio de conveniencia, pero el objetivo fue hallar un grupo de personas sin diagnóstico, pero con alto malestar comparable con el grupo de personas con diagnóstico en base a características observables y controlando por un grupo sin malestar ni diagnóstico. Se identificaron los jóvenes en cada grupo (con diagnóstico, malestar y control) con una probabilidad más próxima (matching). Descontando los missings, los sujetos finalmente entrevistados fueron 105 (con diagnóstico $=37$, malestar $=33$, control $=35$ ) y con una proporción de $31 \%$ hombres y $69 \%$ mujeres. Todos los participantes residían en el territorio catalán, incluyendo zonas rurales. La pérdida de muestra fue debida a cambio de domicilio, imposibilidad de contacto, o negativa a ser entrevistado. Las características sociodemográficas de los missings no fueron significativamente diferentes con respecto a las de los informantes entrevistados.

Este estudio fue aprobado por el Ministerio de Ciencia e Innovación y la Fundació La Marató de TV3 (investigación biomédica) y sus procedimientos evaluados y aprobados por el Comité de Ética de la Fundació Congrés Català de Salut Mental. Todos ellos cumplen los estándares éticos de la Declaración de Helsinki de 1964, incluyendo los referentes al consentimiento informado.

\section{Instrumentos}

Se utilizaron entrevistas semi-estructuradas de tipo cualitativo con el objetivo de explorar las estrategias de los jóvenes de afrontar el malestar de tipo depresivo, incluyendo el uso/evitación de los servicios y el consumo de substancias psico-activas, Las entrevistas se realizaron en castellano o catalán de acuerdo con la lengua materna del entrevistado. Adicionalmente se administraron otros instrumentos validados como la escala MISS (Manheim Interview on Social Support) (Vázquez y Garcia-Boveda, 1997), el BDI-II (BECK-II
Depression Inventory) (Beck, Ward, Mendelson, Mock y Erbaugh, 1961; Sanz, Perdigón y Vázquez, 2003) y el screening de la GHQ (Goldberg Health Questionary) (Goldberg, Bridges, Duncan-Jones, y Grayson, 1988; Monton, Pérez, Campos, García y Lobo, 1993; Rocha, Pérez, Rodríguez-Sanz, Borrell, y Obiols, 2011) para depresión y ansiedad. El número de entrevistadores fue de 11, todos ellos investigadores en antropología médica y/o psicología. Previamente se realizaron dos sesiones con todos los entrevistadores para aunar criterios y coordinar la dinámica de las entrevistas, las cuales se realizaron entre los meses de marzo y octubre de 2011 y a conveniencia de los participantes tras contacto telefónico. Todas las entrevistas fueron heteroadministradas, salvo la parte cuantitativa del cuestionario de drogodependencias que fue autoadministrada.

De forma adicional se realizaron tres grupos focales con algunos jóvenes que habían sido previamente entrevistados (entre cuatro y ocho participantes en cada grupo) con el propósito de triangular y valorar la información cualitativa resultado de la investigación. El guión de los grupos focales incluyó preguntas sobre autoatención/automedicación de los malestares entre los jóvenes. Los grupos focales incluyeron a los tres grupos de nuestra submuestra (con diagnóstico, con malestar autopercibido y control) y diversidad de género y se efectuaron en una sala acondicionada para tal fin en un centro cívico de Barcelona entre abril y junio de 2012.

\section{Variables}

Las variables sobre depresión y malestar emocional se definieron a partir de diferentes parámetros con el fin de controlar la existencia de los síntomas en el pasado y en el momento de realización de la investigación. Concretamente, se valoró la existencia (1) o no (0) de un diagnóstico de depresión/ansiedad en las oleadas $1^{\mathrm{a}}$ y $4^{\mathrm{a}}$ del PFI, así como de malestar emocional asociado y sin diagnóstico en las oleadas $2^{\mathrm{a}}$ y $3^{\mathrm{a}}$, que también fue dicotomizado. La ansiedad y la depresión en la GHQ fueron definidas por su punto de corte y dicotimizadas en términos de depresión o ansiedad versus no existencia de estos síntomas. La condición de BDI media, moderada o severa fue dicotomizada como (1) versus el nivel BDI leve (0).

Las variables sobre consumo de tabaco se definieron a partir de: 1) haber fumado o no haber fumado alguna vez en la vida, 2) fumar actualmente, 3) fumador habitual entendido como fumar cuatro días a la semana o más, 4) fumador diario y 5) fumador en todas las oleadas. Adicionalmente, se preguntó por la edad de inicio del consumo de tabaco y sobre la existencia de esta práctica en el contexto doméstico. Otras variables incluidas fueron la frecuencia de consumo de alcohol y el abuso del mismo en el último año, el consumo de cánnabis en el último año y la existencia de estas prácticas en el contexto familiar. 


\section{Análisis}

Los datos cuantitativos fueron analizados con el software SPSS 20. La información fue codificada e introducida en una base de datos para ser posteriormente fusionada con la base de datos original del Panel de Familias e Infancia, la cual contenía 2416 variables de las precedentes consultas para los 105 sujetos de análisis. En una primera fase se realizó un análisis de consistencia para crear posteriormente nuevas variables que recogieran información tanto de la oleada actual como de las oleadas anteriores. $\mathrm{Al}$ contar con una muestra limitada, se optó por la creación de variables dicotómicas que permitieran realizar el análisis manteniendo un número de sujetos mínimo en cada categoría. Se llevó a cabo estadística descriptiva y bivariada, cálculo de odds ratio, intervalos de confianza y significancia estadística. Finalmente se elaboró un análisis exhaustivo de las principales variables para escoger posteriormente las relaciones estadísticamente más significativas.

Los datos cualitativos fueron gestionados con el software ATLAS.ti 6.2.27 (Computersofware, 1993-2013). Se llevó a cabo un análisis temático inicial para identificar los contenidos principales de los datos obtenidos mediante la discusión colectiva de las observaciones. Seguidamente se organizó una estructura de codificación de acuerdo con los principios de la teoría fundamentada (Glaser y Strauss, 1967) y el método etnográfico, incluyendo el descubrimiento de tipologías nativas o emic. Posteriormente se revisaron todas las transcripciones y se aplicaron los códigos incluyendo la identificación y análisis de las excepciones, así como la reflexividad y la revisión entre pares. Para corroborar tipologías se utilizaron también técnicas de análisis de dominios culturales. Para ello se transformaron algunos datos cualitativos en matrices binarias (sujeto-motivos de fumar). Los datos de dominios culturales fueron analizados con UCINET 6.454 (Borgatti, Everett y Freeman, 2002) mediante un análisis de correspondencia (método: coordenadas principales). Los resultados generales se compararon con los obtenidos de los tres grupos focales.

\section{Resultados}

\section{Características generales de la muestra}

La Tabla 1 muestra las características generales de los sujetos que participaron en la investigación. Los jóvenes tenían entre 17 y 21 años de edad, con una mayoría de mujeres $(68,6 \%)$, puesto que la selección respondió a criterios de diagnóstico/malestar depresivo/ansioso y son ellas el colectivo que usualmente presenta mayor prevalencia e incidencia de los mismos. De forma congruente con los resultados de la explotación de la muestra PFI general (Julià et al. 2012; Martínez-Hernáez et al. 2012), el 45.2\% de los participantes se definió como fumador en el momento de realización del estudio, distribuyéndose en los subgrupos del siguiente modo: el 35\% pertenece al grupo de diagnosticados, el $23 \%$ al de malestares, y el $42 \%$ al de control. El total de casos con BDI severa $(n=5)$ se produjo en el grupo con diagnóstico previo y la mayoría de las situaciones de depresión según el cribado GHQ entre este grupo y el de malestar previo. Llama la atención la presencia de 20 casos de ansiedad según la GHQ entre el grupo control. Asimismo, los sujetos con malestar previo muestran una red social más limitada, tanto en lo que respecta al número de familiares como al de amistades.

\section{Depresión/ansiedad y consumo de tabaco}

El análisis bivariado (Tabla 2) muestra que la probabilidad (OR) de tener una BDI superior a leve es de 3,55 para los que tuvieron un diagnóstico de depresión/ansiedad o malestar autopercibido en las oleadas anteriores. La probabilidad (OR) cuando el punto de corte del BDI es depresión severa y moderada versus media y leve es de 8,543 (IC95\%:1,045-69,82, p=.045). En el caso de depresión según el GHQ, la probabilidad (OR) es de 2,84. La asociación con el GHQ de ansiedad no es significativa, probablemente por el número de casos en el grupo control. Las puntuaciones BDI y de depresión GHQ muestran una alta concordancia (OR: 28,42). No obstante, los resultados indican una asociación negativa entre el consumo de tabaco y la existencia de un diagnóstico o malestar previo de tipo depresivo/ansioso, especialmente para la variable de ser fumador durante todas las oleadas y, en menor medida, $(p<.10)$ para ser fumador actual o haber fumado alguna vez en la vida. Algo parecido ocurre con el abuso de alcohol más de una vez al mes (OR: $0,21)$.

Los resultados tampoco indican una asociación significativa $(p \leq .05)$ entre las diferentes variables de consumo de tabaco y depresión según la escala BDI, salvo cuando realizamos el análisis exclusivamente entre los varones, ya que la probabilidad de ser fumador teniendo un BDI superior a leve es en este caso de 6,22 (IC95\%, 1,06-36,21, p=.042). Adicionalmente, se observan algunas tendencias en el grupo general, pues la probabilidad $(p \leq .10)$ de fumar habitualmente para aquellos que tienen una BDI superior a leve es de 2,22. Sobre la edad de inicio del consumo, su asociación con la BDI es negativa (OR: 0,3) con un valor de $p \leq 0,5$. La medida de ansiedad GHQ, por su parte, muestra una asociación negativa (OR: 0,39$)$ con haber fumado durante todas las oleadas.

Como era previsible, se producen asociaciones significativas entre el consumo de las diferentes substancias psicoactivas. De esta forma, ser fumador de cánnabis en el último año se asocia significativamente con todas las variables de consumo de tabaco y el abuso de alcohol con ser fumador actual y fumar desde la primera oleada. El consumo de tabaco y de cánnabis por parte de otros miembros de la unidad doméstica también se encuentran asociados entre sí. El primero se vincula significativamente con el consumo diario de 
Tabla 1

Muestra

\begin{tabular}{|c|c|c|c|c|c|c|c|c|}
\hline & \multicolumn{6}{|c|}{ Tipo de diagnóstico } & \multirow[b]{3}{*}{ Total } \\
\hline & & \multicolumn{2}{|c|}{ Diagnosticados } & \multicolumn{2}{|c|}{ Malestar } & \multicolumn{2}{|c|}{ Grupo control } & \\
\hline & & $\mathbf{N}$ & Media & $\mathbf{N}$ & Media & $\mathbf{N}$ & Media & \\
\hline \multirow[t]{3}{*}{ Sexo } & Hombre & 13 & & 9 & & 11 & & 33 \\
\hline & Mujer & 24 & & 24 & & 24 & & 72 \\
\hline & Total & 37 & & 33 & & 35 & & 105 \\
\hline \multirow[t]{5}{*}{ Edad } & 17 años & 5 & & 3 & & 7 & & 15 \\
\hline & 18 años & 10 & & 6 & & 5 & & 21 \\
\hline & 19 años & 12 & & 10 & & 9 & & 31 \\
\hline & 20 años & 7 & & 9 & & 12 & & 28 \\
\hline & 21 años o más & 3 & & 5 & & 2 & & 10 \\
\hline \multirow[t]{4}{*}{ Nivel de ingresos familiares } & Menos de $18000 €$ & 6 & & 5 & & 8 & & 19 \\
\hline & De 18001 a $36000 €$ & 18 & & 13 & & 13 & & 44 \\
\hline & $36001 €$ o más & 9 & & 7 & & 5 & & 21 \\
\hline & No recuerda & 4 & & 7 & & 8 & & 19 \\
\hline \multirow[t]{3}{*}{ Estructura familiar año 2010} & Monoparental & 11 & & 7 & & 14 & & 32 \\
\hline & Biparental o reconstituida & 17 & & 19 & & 19 & & 55 \\
\hline & No se dispone de información & 9 & & 7 & & 2 & & 18 \\
\hline \multirow[t]{2}{*}{ Ha fumado alguna vez en su vida } & No ha fumado nunca & 12 & & 11 & & 6 & & 29 \\
\hline & Ha fumado alguna vez & 25 & & 22 & & 29 & & 76 \\
\hline \multirow[t]{2}{*}{ Fumador actual } & No fuma & 18 & & 12 & & 22 & & 52 \\
\hline & Sí fuma & 19 & & 21 & & 13 & & 53 \\
\hline \multirow[t]{2}{*}{ Fumador habitual (4 días o más a la semana) } & No fuma habitualmente & 13 & & 8 & & 16 & & 37 \\
\hline & Sí fuma habitualmente & 24 & & 25 & & 19 & & 68 \\
\hline \multirow[t]{2}{*}{ Fumador diario } & No fuma diariamente & 25 & & 28 & & 21 & & 74 \\
\hline & Sí fuma diariamente & 12 & & 5 & & 14 & & 31 \\
\hline \multirow[t]{4}{*}{ Evolución como fumador en las oleadas } & No ha fumado nunca & 10 & & 11 & & 3 & & 24 \\
\hline & Fumaba antes pero ahora ya no fuma & 9 & & 10 & & 10 & & 29 \\
\hline & Fuma ahora pero no ha fumado siempre & 10 & & 6 & & 6 & & 22 \\
\hline & Ha fumado siempre & 8 & & 6 & & 16 & & 30 \\
\hline \multirow{2}{*}{$\begin{array}{l}\text { Alguna de las personas con quienes convive } \\
\text { fuma }\end{array}$} & No & 16 & & 17 & & 11 & & 44 \\
\hline & $\mathrm{Si}$ & 21 & & 16 & & 24 & & 61 \\
\hline \multirow[t]{2}{*}{ Edad cuando comenzó a fumar } & 14 años o menos & 11 & & 9 & & 14 & & 34 \\
\hline & 15 años o más & 13 & & 10 & & 13 & & 36 \\
\hline \multirow[t]{2}{*}{ Ha fumado cánnabis en el último año } & No ha fumado cánnabis & 17 & & 20 & & 20 & & 57 \\
\hline & Ha fumado cánnabis & 20 & & 13 & & 15 & & 48 \\
\hline \multirow{3}{*}{$\begin{array}{l}\text { Alguna de las personas con quienes convive } \\
\text { fuma (o ha fumadol cánnabis }\end{array}$} & No & 23 & & 24 & & 19 & & 66 \\
\hline & Ha fumado, pero ahora no fuma. & 8 & & 5 & & 11 & & 24 \\
\hline & Si, fuma actualmente & 6 & & 2 & & 5 & & 13 \\
\hline Frecuencia de abuso de alcohol en el último año & Ninguna vez & 7 & & 4 & & 5 & & 16 \\
\hline & 1 vez al mes o menos frecuencia & 19 & & 24 & & 11 & & 54 \\
\hline & Más frecuentemente & 11 & & 5 & & 19 & & 35 \\
\hline Nivel de BDI & Leve & 20 & & 24 & & 30 & & 74 \\
\hline & Medio & 8 & & 4 & & 3 & & 15 \\
\hline & Moderado & 4 & & 5 & & 2 & & 11 \\
\hline & Severo & 5 & & 0 & & 0 & & 5 \\
\hline Nivel de ansiedad Goldberg & No ansiedad & 10 & & 9 & & 15 & & 34 \\
\hline & Si ansiedad & 27 & & 24 & & 20 & & 71 \\
\hline Nivel de depresión Goldberg & No depresión & 8 & & 8 & & 17 & & 33 \\
\hline & Si depresión & 29 & & 25 & & 18 & & 72 \\
\hline Manheim. Número total familiares & & & 5,1 & & 4,2 & & 4,9 & \\
\hline Manheim. Número total amigos & & & 8,8 & & 8,2 & & 8,8 & \\
\hline
\end{tabular}

tabaco de los sujetos. El segundo con el consumo habitual y diario de tabaco, así como con el consumo de cánnabis.

Cuando se realiza un análisis bivariado exclusivamente para mujeres, los resultados no revelan una asociación significativa entre las variables de consumo de tabaco y las de de- presión/ansiedad. Sin embargo, esta relación sí se produce entre el consumo de otras substancias, pues la probabilidad (OR) de haber fumado cánnabis en el último año siendo fumadora de tabaco actualmente es de 5,1 (IC 95\% 1,891$14,062 p=.001$ ) y de abusar de alcohol de 3,5 (IC 95\%: 1,275- 


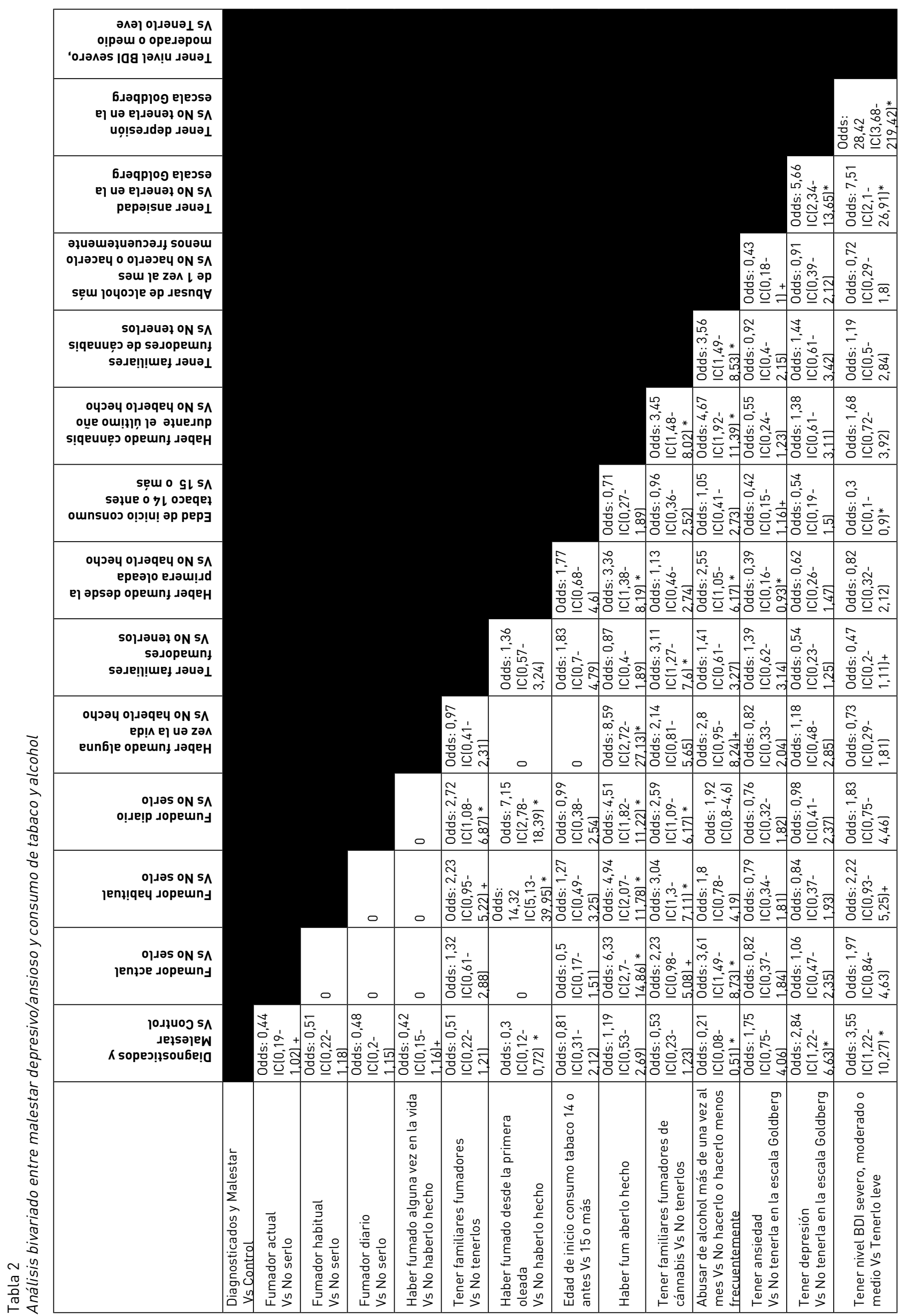


$10,014, p=.015)$. Asimismo, la probabilidad de fumar tabaco habitualmente teniendo consumidores en el hogar es de 5,9 (IC $95 \%$ 1,754-19,904, $p=.004$ ). Las jóvenes que han fumado tabaco desde la primera oleada tienen una OR de 3,3 (IC $95 \% 1,151-9,655 p=.027)$ de abusar de alcohol. Por su parte, el consumo de cánnabis en el último año se asocia con abuso de alcohol (OR: 4,750 IC 95\% 1,642-13,740 p=.004) y con la existencia de consumidores de esta misma substancia en el hogar (OR 3,107 IC 95\% 1,074-8,993 p=.037).

\section{Ítems del BDI-II y consumo de tabaco}

$\mathrm{Si}$ analizamos la asociación entre los diferentes ítems de la escala BDI con la existencia de un diagnóstico o malestar previo (Tabla 3), observamos que son significativos a $\mathrm{p} \leq 0,05$ tener tristeza, sentimiento de autodesprecio, llanto, falta de energía, cambio de apetito y dificultades de concentración. En el grupo de mujeres, se añaden los ítems "pesimismo" (OR: 3,4 IC 95\%: 1,189-9,723 $p=.022$ ), sentimiento de castigo (OR 5,5 IC 95\% 1,148-26,357 p=.033) y crítica (OR 3,7 IC $95 \%$ 1,29-10,62 $p=.01)$. En cambio, el sentimiento de autodesprecio no resulta significativo. Por otro lado, en el grupo de hombres, el único ítem que guarda una asociación significativa con haber padecido previamente una depresión/ansiedad diagnosticada o un malestar autopercibido es "falta de energía" (OR: 7,8 IC 95\% 1,35-45,83 p=.02).

En cuanto a la relación entre consumo de tabaco y los ítems BDI, se observa que "falta de interés" resulta significativo para ser fumador de tabaco actual y habitual y que casi en el límite $(p=.053)$ emerge una asociación inversa entre falta de placer y haber sido fumador durante todas las oleadas. No obstante, si realizamos el análisis bivariado separadamente en mujeres y hombres aparecen algunos datos significativos:

Entre las jóvenes, fumar habitualmente se asocia con falta de interés (OR 2,778 IC 95\% 1,01-7,64 p=.048) y fumar diariamente con indecisión (OR 3,043 IC 95\% 1,107-9,104 $p=.046)$. Ser fumadora actual, por su parte, se asocia negativamente con "crítica" (OR 0,360 IC 95\% 0,138-0,934 p=.036) y haber fumado siempre, de forma también negativa, con falta de placer (OR 0,300 IC 95\% 0,096-0,944 p=.039).

Entre los jóvenes, el cansancio y la fatiga se asocia con el consumo de tabaco (OR 16,8 IC 95\% 2,74-102,8 p=.002), con ser fumador habitual (OR 6,75 IC 95\% 1,42-31,89 p=.016), fumador diario (OR 5,33 IC 95\% 1,05-26,89 $p=.043$ ) y, en menor medida, con haber fumado durante todas las oleadas (OR 4,0 IC 95\% 0,78-20,31 p=.095). Adicionalmente, la probabilidad de sentir "falta de interés" y "pesimismo" entre los fumadores es de 5,28 (IC 95\% 1,11-23,31 $p=.028$ ) y de 4,87 (IC95\% 1,08-23,56, p=.049) respectivamente. Los "pensamientos suicidas", por su parte, se asocian con el consumo habitual (OR: 11,87, IC 95\%: 1,19-118,49, $p=.035$ ) y el consumo diario de tabaco (OR 7, IC 95\% 1,02-47,96 p=.048).

\section{Ítems del screening GHQ y consumo de tabaco}

Los ítems de la escala GHQ que se asociaron con la existencia previa de un diagnóstico o malestar fueron "sufrir temblores, hormigueos, mareos, sudores y/o diarrea" (OR: 3,62 IC 95\%: 1,21-10,80 p=.021), junto con "sentirse con poca energía. (OR: 3,04, IC 95\%: 1,31-7,07, $p=.010$ ) y "perder el interés por las cosas" (OR: 3,33, IC 95\%: 1,14$9,67, p=.027)$. Entre las mujeres se añadieron "tener dolores de cabeza o nuca" (OR: 3,00, IC 95\%:1,01-8,90, p=.048) y la asociación inversa con despertarse demasiado temprano (OR: 0,22, IC 95\%: 0,060-0,824, p=.024). Entre los hombres ningún ítem guardó una asociación estadísticamente significativa con tener un diagnóstico/malestar previo.

Cuando analizamos la asociación entre el consumo de tabaco y los diferentes ítems de la escala GHQ, observamos que la única significativa es la relación inversa con "sufrir dolores de cabeza o nuca”, con una pauta semejante para todas las variables vinculadas al uso del tabaco, con la excepción del consumo diario; esto es: fumar actualmente (OR: 0,30, IC95\%: 0,12-0,72, $p=.007$ ), fumar habitualmente (OR: $0,31$, IC 95\%:0,12-0,78, $p=.013)$, haber fumado siempre (OR: 0,34, IC 95\%: 0,13-0,92, p=.03) o haber fumado alguna vez en la vida (OR: 0,33, IC 95\%: 0,12-0,88, $p=.027$ ).

Entre los varones destaca la asociación entre fumar habitualmente y "sufrir temblores, hormigueos, mareos, sudores y/o diarrea” (OR: 6,00, IC 95: 1,03-35,90, p=.05); también entre ser fumador y "estar preocupado por la propia salud" (OR: 10 IC95\%: 0,99-100,46, $p=.05$ ) y ser fumador y "sentirse desesperanzado" (OR: 10,50 IC95\%: 1,11-98,91, p=.04). Entre ellas destacan, además de la ya citada asociación inversa con "sufrir dolores de cabeza o nuca", las también probabilidades negativas entre ser fumador y "perder la confianza en sí mismo" (OR: 0,22, IC 95\%: 0,06-0,79, p=.02), entre haber fumado desde siempre y la "preocupación por la propia salud” (OR: 0,10 IC 95\%: 0,01-0,82, p=.03), así como la asociación positiva entre fumar habitualmente y "perder peso por causa de la pérdida del apetito" (OR: 4,52, IC95\%: 1,11$18,28, p=.03)$.

\section{Resultados del análisis cualitativo}

Los resultados de la investigación cualitativa con los 105 participantes ofrecen información principalmente sobre los motivos percibidos de consumo de tabaco. Tras el análisis de las diferentes causas verbalizadas por los informantes se realizó una codificación, corroborada mediante los grupos focales, según los siguientes tipos:

1) Automedicación. En este conjunto de motivos se incluyen los usos asociados con el auto-cuidado del malestar por parte de los consumidores. Se indica que fumar "relaja", "resuelve la ansiedad", "calma los nervios" o permite "desahogarse”. Estas motivaciones permiten la persistencia del consumo o se plantean, en otras ocasiones, como episódicas: "He decidido volver [a fumar] por una mala época después de haberlo dejado. Tengo intención de volverlo a dejar”. Este tipo es el más frecuente entre los fumadores, 


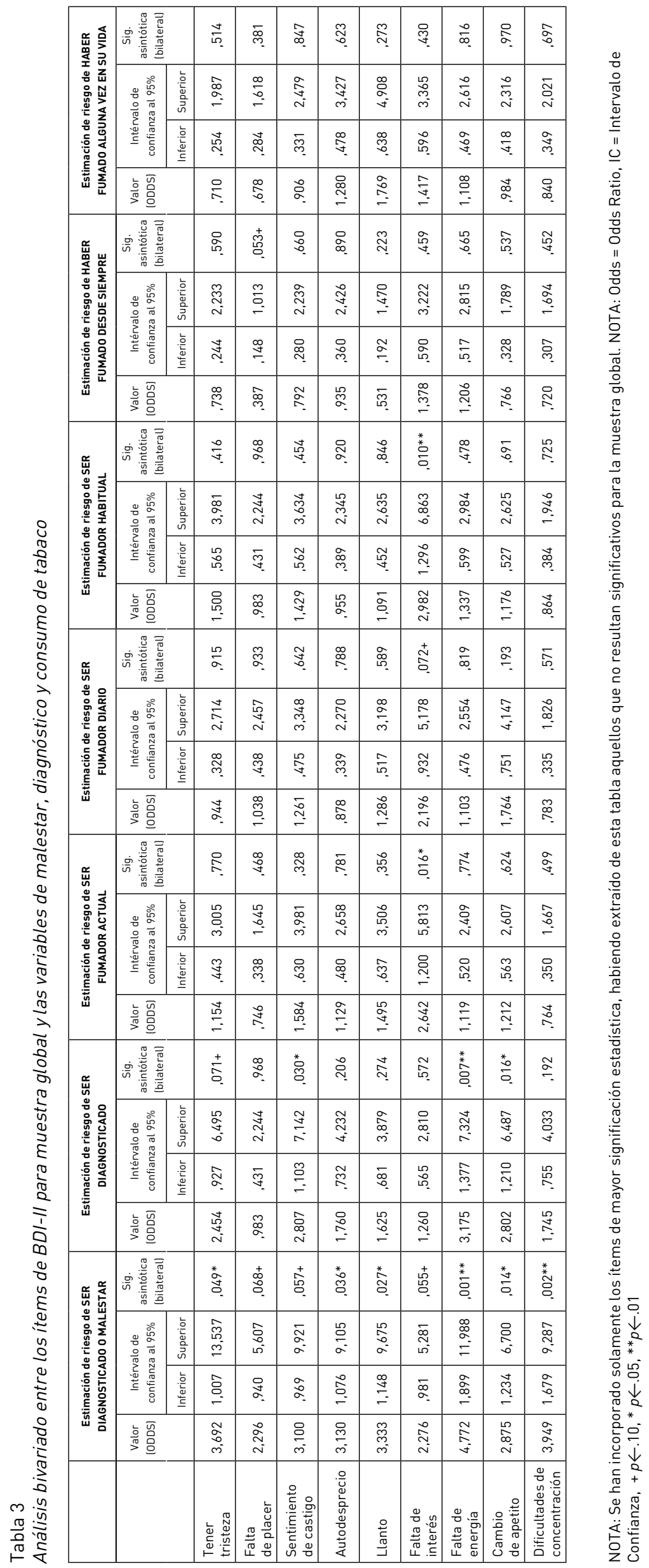

tanto hombres como mujeres, con diagnóstico previo de depresión/ansiedad.

2) Hábito. En este tipo se incluyen las razones asociadas a la costumbre y normalización del hábito tabáquico y resulta más frecuente en la verbalización de los fumadores con malestar previo, especialmente las jóvenes.

3) Experimentación. En este grupo se incluyen las razones asociadas al consumo ocasional. Este momento es ubicado por los informantes alrededor de los 14 o 15 años. Este motivo fue el más citado por las fumadoras con malestar previo.

4) Influencia del entorno. La importancia del contexto y de la red social de amistades es principalmente citada por los fumadores que dan testimonio de situaciones como: "empecé a fumar para no ser menos que mis amigos", "porque me ofrecieron", "para acompañar a mis amigos cuando fuman", "porque a medida que fui creciendo la tolerancia al tabaco era mayor en mi entorno" o "porque mis amigos fuman", entre otros. El entorno familiar también destaca por su influencia: "Cuando está mal [mi madre] se queda fumando toda la tarde en el sofá". Los que más se sienten influidos por el entorno son los fumadores con malestar previo.

5) Adicción. En este tipo se incluyen verbalizaciones como "Estoy enganchado", "No puedo dejarlo", "Es un vicio" o "Por adicción”. El grupo más destacado en este punto son los fumadores control.

6) Placer. En última instancia aparecen los motivos asociados al disfrute derivado de fumar, que son verbalizados como "Me gusta" o "Me da placer". Las fumadoras del grupo control manifiestan más frecuentemente esta opción.

La Figura 1 muestra sintéticamente la asociación entre los diferentes motivos y subgrupos a partir de la transformación de los datos cualitativos en una matriz numérica y la realización con ésta de un análisis de correspondencia. Llama la atención que entre el grupo de fumadores con diagnóstico/malestar previo que destacan la automedicación como motivo de fumar se concentra el $60 \%$ de sujetos con BDI severo. 


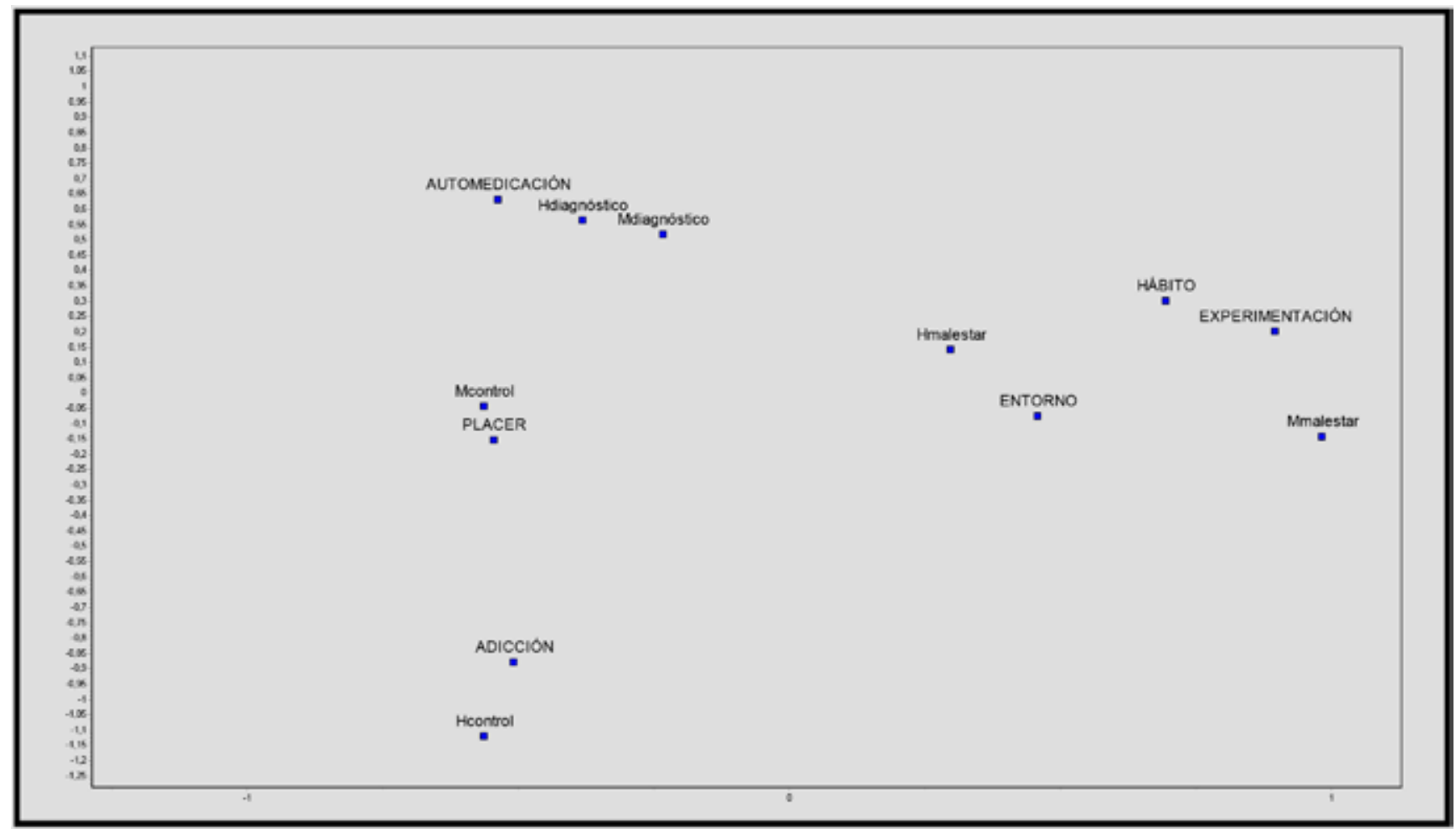

Figura 1. Análisis de correspondencia de los motivos de fumar y los subgrupos de participantes

\section{Discusión}

Este estudio revela que los jóvenes de esta muestra con depresión y/o ansiedad se inician más tardíamente al consumo de tabaco y que, con la excepción de los varones, no fuman en mayor proporción que los sujetos que no padecen estos malestares, pero cuando fuman lo hacen principalmente para automedicar sus síntomas. Adicionalmente, el consumo de tabaco se asocia en varias de sus modalidades con el abuso de alcohol, el consumo de cánnabis, la existencia de fumadores en el hogar y de consumidores de cánnabis, mostrando un panorama de policonsumo e influencias de la red social confirmadas en la literatura (Guo et al., 2010), (Emory et al., 2010), (Turner, Mermelstein y Flay, 2004), (Alcalá, Azañas, Moreno y Gálvez, 2002), (Peters, Budney y Carroll, 2012). No obstante, los resultados no están exentos de paradojas. Por ejemplo, en nuestro estudio el consumo de tabaco y la existencia previa de depresión/ansiedad o de malestar del mismo espectro no aparecen asociados entre sí o lo hacen de forma negativa. Es evidente que la existencia de diagnóstico o malestar previo no significa necesariamente sufrir depresión o ansiedad en el momento de realización del estudio, pues aunque algunos casos de estos trastornos puedan cronificarse, también los mismos responden a cierta fluctuación relacionada con los procesos vitales o adquieren una expresión clínica episódica que puede no ser capturada en el momento de la entrevista. Sin embargo, la asociación entre BDI severo/medio con diagnóstico/malestar previo
(OR: 8,543, IC95\%:1,045-69,82, $p=.045$ ) hace pensar en otras variables en juego. Una de ellas es, probablemente, que la supervisión parental en estos casos permite un acceso más tardío y limitado al consumo de tabaco y de otras substancias psicoactivas por parte de los jóvenes. Este argumento es congruente con datos de este mismo estudio (ver Tabla 2), como la asociación negativa entre diagnóstico/malestar previo y consumo de tabaco desde la primera oleada (OR: 0.3). En realidad, el grueso de los sujetos no fumadores se encuentra en los grupos de diagnóstico y malestar (ver Tabla 1). Algunas investigaciones en el mismo ámbito y con la muestra general del Panel de Familias e Infancia (PFI) han mostrado que, entre los adolescentes que sufren de estados de ánimo negativos, la comunicación intergeneracional atenúa el riesgo de consumo diario (Martínez-Hernáez et al., 2012) y el consumo semanal de tabaco (Julià, Escapa, Marí-Klose y Marí-Klose, 2012). De esta forma, los sujetos con diagnóstico/malestar previo podrían fumar menos debido a la protección parental por causa de su mayor vulnerabilidad y, quizá, por su incorporación más tardía o no incorporación a redes sociales de iguales donde el consumo de tabaco es más prevalente.

En congruencia con lo que acabamos de indicar, observamos que el BDI superior a leve se asocia negativamente con la edad de inicio al consumo de tabaco (OR: 0.3). De esta forma, los afectados por síntomas depresivos, la mayoría de los cuales tenía un diagnóstico o malestar previo, o no fu- 
man o se han incorporado más tarde al hábito tabáquico. Sin embargo, entre ellos existe una asociación con ser fumador habitual (OR: 2,22) en el momento de realización de este estudio, aunque con una significación $p \leq .10$, debido con toda probabilidad al tamaño de la muestra. Adicionalmente, entre los varones ser fumador sí que se asocia de forma significativa (OR: 6,22, IC95\%, 1,06-36,21, $p=.042$ ) con el nivel BDI superior a leve, aunque no para otras medidas del consumo como el habitual o el diario. Es probable que la mayor popularización del consumo de tabaco entre las jóvenes tenga algo que ver con la ausencia de esta asociación entre las mujeres. La diferenciación del consumo por género entre adolescentes es conocida por estudios anteriores (Mendoza, López y Sagrera, 2007). Encuestas recientes (ESTUDES, 2010) muestran resultados en el mismo sentido al evidenciar que el 43,8\% de las chicas entre 14 y 18 años ha probado alguna vez el tabaco frente al 35,6\% de los chicos.

Los datos cualitativos nos revelan que los fumadores con depresión/ansiedad previa fuman principalmente como recurso de automedicación. Este dato es congruente con algunas aportaciones recientes de laboratorio (Kassel et al., 2007) que indican que fumar cigarrillos reduce el afecto negativo entre los adolescentes que tienen una fuerte convicción de que fumar alivia sus malestares. De esta forma, y como han indicado otros autores (Audrain-McGovern, et al., 2009), los síntomas depresivos pueden promover el consumo de tabaco y éste reducir a su vez la depresión, principalmente cuando existe una autopercepción de que fumar será efectivo.

Este estudio presenta varias limitaciones a ser consideradas. La primera de ellas es el tamaño de la muestra que ha impedido controlar el efecto de otros factores sobre la asociación entre las variables de consumo de tabaco y de depresión/ansiedad. Por otro lado, no se estableció una medida sobre dependencia nicotínica, que hubiese podido establecer una diferencia entre fumadores, principalmente para dar cuenta de si los casos de fumadores con depresión/ ansiedad previa que alegan motivos de automedicación son también dependientes nicotínicos. Piénsese que algunos estudios indican que el éxito de deshabituación tabáquica es menor entre los afectados por una depresión mayor de larga evolución (Weinberger et al., 2011). Otra limitación es la no exclusión de los casos con ansiedad según la GHQ en el grupo control; no obstante, el objetivo principal del diseño era valorar la asociación entre depresión/ansiedad en oleadas anteriores y el consumo de tabaco.

Adicionalmente, los resultados de esta investigación muestran una mayor prevalencia de consumo de tabaco entre los participantes $(45,2 \%)$ en comparación con los datos recientes de la Enquesta de Salut de Catalunya (ESCA) 2012, que indica tasas de $30,5 \%$ y de $28 \%$ de fumadores diarios y ocasionales para los varones y mujeres, respectivamente, en la franja etaria de 15-24 años. Sin embargo, esta discrepancia se presenta también en la comparación entre los datos de la ESCA y los derivados de la explotación general del PFI. En un estudio reciente con los datos de la tercera oleada del PFI (Julia et al. 2012), se indica que el 55,3\% había fumado alguna vez tabaco y que el $53,2 \%$ había fumado en los últimos treinta días; concretamente: $16,3 \%$ menos de una vez a la semana, $8,2 \%$ uno o dos días a la semana, $4,9 \%$ tres o cuatros días a la semana, 3,3\% cinco o seis días a la semana y 20,5\% cada día. En la medida en que nuestro estudio se realiza seleccionando una submuestra del PFI a partir de los criterios ya indicados de haber recibido un diagnóstico de depresión/ansiedad o haber expresado malestar autopercibido en anteriores oleadas del PFI, es lógico que la prevalencia sea más cercana a los resultados del PFI que de la ESCA. Probablemente, esta disparidad es consecuencia de la aplicación de modelos metodológicos diferentes a la hora de valorar el consumo de tabaco, especialmente el consumo ocasional.

A pesar de las limitaciones indicadas, el estudio ofrece una integración de metodologías y resultados que puede ser útil para investigaciones posteriores que analicen el papel de los motivos autopercibidos de automedicación entre los fumadores con depresión/ansiedad, así como para que las campañas de deshabituación tabáquica dirigidas a los jóvenes consideren la importancia de las relaciones entre tabaco y salud mental.

\section{Reconocimientos}

Esta investigación ha sido financiada por la Fundació Marató TV3 (Proyecto: The emotional distress of adolescents: lifestyles, mental health and lay strategies used in managing adversity, n. ${ }^{\circ}$ identificación $090730 / 31$ ) y por el MICINN (Proyecto Los malestares de los adolescentes, CSO200908432 y CSO2012-33841). Agradecemos a los jóvenes que participaron en el estudio su colaboración y entusiasmo con la investigación. También a los entrevistadores Patricia Vigueras, Maria Antonia Monserrat, Laia Pibernat, Carlos Piñones, Federico Montseny, Marta Farré, Mireia Castro y Eva Marxen.

\section{Conflictos de intereses}

No existen conflictos de intereses.

\section{Referencias}

ATLAS.ti. Qualitative data analisys. Version 6.2.27. [Computersofware] (1993-2013) Berlin. ScientificSofwareDevelopment.

Alcalá, M., Azañas, S., Moreno, C. y Gálvez, L. (2002). Consumo de alcohol, tabaco y otras drogas en adolescentes, estudio de dos cortes. Medicina de Familia (Andalucía), 3, 81-87. 
Audrain-McGovern, J., Rodriguez, D. y Kassel, J. D. (2009). Adolescent smoking and depression: evidence for self-medication and peer smoking mediation. Addiction, 104, 1743-1756. doi: 10.1111/j.1360-0443.2009.02617.x.

Balázs, J., Miklosi, M., Kereszteny, A., Apter, A, Avigal S, Bobes J,...Wasserman D. (2013). Adolescent Subthreshold-Depression and Anxiety: Psychopathology, Functional Impairment and Increased Suicide Risk. Journal of Child Psychology and Psychiatry. doi: 10.1111/jcpp.12016.

Beck, A. T., Ward, C. H., Mendelson, M., Mock, J. y Erbaugh, J. (1961). An Inventory for Measuring Depression. Archives of General Psychiatry, 4, 561-571. doi:10.1001/archpsyc.1961.01710120031004.

Birchwood, M. y Singh, S. P. (2013). Mental health services for young people: matching the service to the need. British Journal of Psychiatry Supplements, 54:s1-2. doi: 10.1192/bjp.bp.112.119149.

Borgatti S. P., Everett M. G. y Freeman. L. C. (2002). Ucinet for Windows: Software for Social Network Analysis. Harvard, MA: Analytic Technologies.

Calleja, N. y Aguilar, J. (2008). Por qué fuman las adolescentes: un modelo estructural de la intención de fumar. Adicciones, 20, 387-394.

Chaiton M. O., Cohen J. E., O’Loughlin J. y Rehm J. (2009). A systematic review of longitudinal studies on the association between depression and smoking in adolescents. BioMed Central Public Health. 9, 356. doi:10.1186/14712458-9-356.

Choi, W. S., Patten, C. A., Gillin, J. C., Kaplan, R. M. y Pierce, J. P. (1997). Cigarette smoking predicts development of depressive symptoms among U.S. adolescents. Annals of Behavioral Medicine, 19, 42-50.

Emory, K., Saquib, N., Gilpin, E. A. y Pierce, J. P. (2010). The association between home smoking restrictions and youth smoking behaviour: a review. Tobacco Control, 19, 495-506. doi:10.1136/tc.2010.035998

Escobedo, L. G., Reddy, M. y Giovino, G. A. (1998). The relationship between depressive symptoms and cigarette smoking in US adolescents. Addiction, 93, 433-440.

Fergusson, D. M., Goodwin, R. D. y Horwood, L. J. (2003). Major depression and cigarette smoking: results of a 21year longitudinal study. Psychological Medicine, 33, 13571367. doi: 10.1017/S0033291703008596.

Fergusson, D., Horwood, J. y Boden, J. (2007). Childhood social disadvantage and smoking in adulthood: results of a 25-year longitudinal study. Addiction, 102, 475-482. doi:10.1111/j.1360-0443.2006.01729.x

Generalitat de Catalunya. (2013). Enquesta de Salut de Catalunya 2010-2014. Barcelona: Departament de Salut.

Glaser, B. G. y Strauss A. L. (1967). The Discovery of Grounded Theory; Strategies for Qualitative Research. Chicago; IL: Aldine Pub Co.
Goldberg, D., Bridges, K., Duncan-Jones, P.y Grayson, D. (1988). Detecting anxiety and depression in general medical settings. British Medical Journal, 297, 897-899.

Guo, H. J., McGee, R., Reeder, T., y Gray, A. (2010). Smoking behaviours and contextual influences on adolescent nicotine dependence. Australian and New Zealand Journal of Public Health, 34, 502-507. doi: 10.1111/j.17536405.2010.00597.x.

Hrubá, D. y Zaloudíková, I. (2010). Why to smoke? Why not to smoke? Major reasons for children's decisions on whether or not to smoke. Central European Journal of Public Health, 18, 202-208.

Institut d' Infància i Mon Urbà. Panel de Familias e Infancia. Barcelona. Recuperado de http://www.ciimu.org/images/stories/CIIMU/Estudios e Investigaciones/ 2panel-fam/14presentacio cggranell.pdf (Consultado en: $19 / 11 / 2012)$.

Julià, A., Escapa, S., Marí-Klose, M. y Marí-Klose, P. (2012). Factores de riesgo psicosociales en el consumo de tabaco de los adolescentes: estados de ánimo negativos, grupo de iguales y estilos parentales. Adicciones, 24, 309-318.

Kassel, J. D., Evatt, D.P., Greenstein, J. E., Wardle, M. C., Yates, M. C. y Veilleux, J. C. (2007). The Acute Effects of Nicotine on Positive and Negative Affect in Adolescent Smokers. Journal of Abnormal Psychology, 116, 543-553. doi: 10.1037/0021-843X.116.3.543

Klungsoyr, O., Nygard, J., Sorensen, T. y Sandanger, I. (2006). Cigarette smoking and incidence of first depressive episode: an 11-year, population-based follow-up study. American Journal of Epidemiology, 163, 421-432.

Lyons, M., Hitsman, B., Xian, H., Panizzon, M. S., Jerskey, B. A., Santangelo, S.,... Tsuang, M. T. (2008). A twin study of smoking, nicotine dependence, and major depression in men. Nicotine E Tobacco Research, 10, 97-108. doi: 10.1080/14622200701705332.

Martínez-Hernáez, Á., Marí-Klose, M., Julià, A., Escapa, S., Marí-Klose, P. y DiGiacomo, S. (2012). Adolescent daily smoking, negative mood-states and the role of family communication. Gaceta Sanitaria, 26, 421-428. doi:10.1016/j.gaceta.2011.09.030

Mendoza, R., López, P. y Sagrera, M. S. (2007). Diferencias de género en la evolución del tabaquismo adolescente en España (1986-2002). Adicciones, 19, 273-288.

Merikangas, K. R., He, J., Burstein, M., Swanson, S. A., Avenevoli, S., Cui, L.,... Swendsen, J. (2010). Lifetime Prevalence of Mental Disorders in U.S. Adolescents: Results from the National Comorbidity Survey ReplicationAdolescent Supplement (NCS-A). Journal of the American Academy of Child Eं Adolescent Psychiatry, 49, 980-989. doi: 10.1016/j.jaac.2010.05.017.

Mineur, Y. S. y Picciotto, M. R. Nicotine receptors and depression: revisiting and revising the cholinergic hypothesis of depression. (2010). Trends in Pharmacological Sciences, 31, 580-586. doi: 10.1016/j.tips.2010.09.004 
Ministerio de Sanidad y Políticas Sociales. (2010). Encuesta Estatal sobre Uso de Drogas en Estudiantes de Enseñanzas Secundarias (ESTUDES). Madrid: Plan Nacional sobre Drogas.

Montón, C., Pérez Echevarría, M. J., Campos, R., García Campayo, J. y Lobo, A. (1993). Escalas de ansiedad y depresión de Goldberg: una guía de entrevista eficaz para la detección del malestar psíquico. Atención Primaria, 12, 345-349.

Peters, E. N., Budney, A. J. y Carroll, K. M. (2012). Clinical correlates of co-occurring cannabis and tobacco use: a systematic review. Addiction, 107, 1404-1417. doi:10.1111/j.1360-0443.2012.03843.x

Rohde, P., Lewinsohn, P. M., Brown, R. A., Gau, J. M. y Kahler, C. W. (2003). Psychiatric disorders, familial factors and cigarette smoking: I. Associations with smoking initiation. Nicotine E Tobacco Research, 5, 85-98.

Rocha, K. B., Pérez, K., Rodríguez-Sanz, M., Borrell, C. y Obiols, J. E. (2011). Propiedades psicométricas y valores normativos del General Health Questionnaire (GHQ12) en población general española. International Journal of Clinical and Health Psychology, 11, 125-139

Rubinstein, M.L., Luks, T.L., Moscicki, A.B., Dryden, W., Rait, M.A. y Simpson G.V. (2011). Smoking-cue induced brain activation in adolescent light smokers. Journal of Adolescent Health, 48, 7-12. doi:10.1016/j.jadohealth.2010.09.016.
Sanz, J., Perdigón, A. L., Vázquez, C. (2003). Adaptación española del Inventario para la Depresión de Beck-II (BDI-II): 2. Propiedades psicométricas en población general. Clínica y Salud, 14, 249-280.

Steuber, T. L. y Danner, F. (2006). Adolescent smoking and depression: which comes first? Addictive Behaviors, 31, 133-136.

Turner, L., Mermelstein, R. y Flay, B. (2004). Individual and contextual influences on adolescent smoking. $A n-$ nals of the New York Academy of Sciences, 1021, 175-197.

Vázquez, A. J. y Garcia-Boveda, R. J. (1997). The Mannheim interview on social support psychometric characteristics of a Spanish version. Social Psychiatry and Psychiatric Epidemiology, 32, 208-214.

Weinberger, A. H., McKee, S. A., Picciotto, M. R. y Mazure, C. M. (2011). Examining antidepressant drug response by smoking status: why is it important and how often is it done? Journal of Psychopharmacology, 25, 1269-76. doi: $10.1177 / 0269881110389347$. 